This item was submitted to Loughborough's Research Repository by the author.

Items in Figshare are protected by copyright, with all rights reserved, unless otherwise indicated.

\title{
Complex potential by hydrodynamic analogy for the determination of flexure- torsion induced stresses in De Saint Venant beams with boundary singularities
}

\section{PLEASE CITE THE PUBLISHED VERSION}

http://dx.doi.org/10.1016/j.enganabound.2013.09.008

\section{PUBLISHER}

(C) Elsevier

\section{VERSION}

AM (Accepted Manuscript)

\section{PUBLISHER STATEMENT}

This work is made available according to the conditions of the Creative Commons Attribution-NonCommercialNoDerivatives 4.0 International (CC BY-NC-ND 4.0) licence. Full details of this licence are available at: https://creativecommons.org/licenses/by-nc-nd/4.0/

\section{LICENCE}

CC BY-NC-ND 4.0

\section{REPOSITORY RECORD}

Barone, Giorgio, Francesco Lo lacono, and Giacomo Navarra. 2019. "Complex Potential by Hydrodynamic Analogy for the Determination of Flexure-torsion Induced Stresses in De Saint Venant Beams with Boundary Singularities". figshare. https://hdl.handle.net/2134/16759. 


\title{
Complex potential by hydrodynamic analogy for the determination of flexure-torsion induced stresses in De Saint Venant beams with boundary singularities
}

\author{
Giorgio Barone ${ }^{1 *}$, Francesco Lo Iacono ${ }^{2}$ and Giacomo Navarra ${ }^{3}$
}

\begin{abstract}
In this paper, a novel complex potential function for the solution of the flexure-torsion problem in De Saint Venant beams is proposed, considering the simultaneous presence of external shear and torsion excitations. By defining a fictitious vector field and taking advantage of a hydrodynamic analogy, the proposed complex potential function allows the stress vector field and the unitary twist rotation of the cross-section to be determined at once, and, therefore, returns the complete solution of the problem. The proposed approach is wellsuited for domains having boundary singularities. A numerical application, implemented by using the Complex Variable Boundary Element Method (CVBEM), is reported for an elliptical cross-section to show the validity of the proposed complex potential. Finally, two singularity problems are analyzed, considering an L-shaped and an epicycloid-shaped crosssection.
\end{abstract}

Author keywords: complex potential; shear; torsion; De Saint Venant beam; boundary singularities.

\footnotetext{
${ }^{1}$ Research Associate, Department of Civil and Environmental Engineering, Engineering Research Center for Advanced Technology for Large Structural Systems (ATLSS Center), Lehigh University, 117 ATLSS Dr., Bethlehem, PA 18015-4729, USA, ingbarone@gmail.com, *Corresponding Author.

${ }^{2}$ Research Associate, Facoltà di Ingegneria ed Architettura, Università degli studi di Enna "Kore", Cittadella Universitaria, 94100 Enna, Italy, francesco.loiacono@unikore.it.

${ }^{3}$ Assistant Professor, Facoltà di Ingegneria ed Architettura, Università degli studi di Enna "Kore”, Cittadella Universitaria, 94100 Enna, Italy, giacomo.navarra@unikore.it.
} 


\section{Introduction}

The determination of the stress distribution in De Saint Venant beams has been extensively studied during the last century, using either stresses, displacements or mixed methods. However, analytical solutions describing the shear stress vector field induced by external flexure-torsion excitations have been provided only for limited cross-section geometries ([1] - [3]). Shear and torsion problems require, for most domains, numerical approaches, the most commonly used being the Finite Element Method (FEM) ([4] - [7]) and Boundary Element Method (BEM) ([8] - [10]). In the first case, since the whole domain needs to be discretized, an inappropriate meshing process can cause loss of accuracy for particularly irregular crosssectional geometries. Moreover, a large number of elements is normally required to achieve a reasonable accuracy for complex profiles, leading to low computational efficiency. The BEM requires a lower number of elements, since only the boundary has to be discretized, and it leads to accurate results with reduced computational effort.

A different strategy is to formulate the problem as a Laplace equation in terms of stress potential function, obtaining either a Dirichlet or a Neumann boundary value problem. For the pure torsion case, the use of a complex potential defined in terms of the so-called Prandtl stress function is well-established ([2], [3], [11]). Analytic solutions for simple domains can be found by conformal mapping methods. For more complex cross-sectional shapes, complex variable numerical methods can be adopted, as, for example, the Complex Polynomial Method (CPM), the Complex Variable Element Method (CVBEM) and the Line Element-less Method ([12] - [15]), among others.

For the case of beams subjected to both torsion and shear, a modified potential stress approach has been proposed in [7], superimposing the stress field generated by the shear forces applied at the flexure center and the stress field due to pure torsion. The two fields are determined by the solution of two Neumann problems and three Dirichlet problems, respectively. An alternative complex potential function, related directly to the shear stresses, has been proposed for the first time in [16], allowing the determination of the complete stress field distribution and unitary twist rotation at once by the LEM, using only line integrals 
without requiring meshing process neither of the domain or the boundary. The use of this complex potential has been extended to the CVBEM in [17] for the case of pure torsion, and in [18] for the case of shear and torsion at once. Moreover, by using a suitable coordinates transformation, the complex potential approach has been successfully applied to orthotropic beams by using LEM in [1][19], [20].

In this paper, a modified complex potential function is introduced, allowing the determination of the stress vector field and the unitary twist rotation at once (and, hence, the torsional rigidity of the cross-section as well). The flexure-torsion problem is formulated as a Dirichlet boundary problem, to be solved in conjunction with the static equivalence condition between the external torsional moment and the internal moment produced by the shear stresses. Due to the particular definition of the proposed complex potential function, the static equivalence conditions with respect to the external shear forces are automatically satisfied, reducing the number of equations needed with respect to the formulation proposed in [16] [18]. Moreover, the proposed complex potential can be used straightforwardly for crosssections having boundary singularities, unlike the previous formulations. The complete solution of the flexure-torsion problem is achieved by only algebraic equations and lineintegrals along the boundary, avoiding any double integral to be performed over the domain, hence returning a pure boundary method. The proposed approach is illustrated through three numerical examples by using the CVBEM. In particular, first, an elliptical cross-section, whose exact solution is known in analytical form, is analyzed to validate the method. Then, an L-shaped and an epicycloidal-shaped cross-section are analyzed to highlight the stability of the method in presence of boundary singularity points.

\section{Torsion and shear: governing equations}

In this section, the well-established De Saint Venant beam theory for isotropic linear elastic materials is briefly introduced, providing the basic equations needed for the flexure-torsion potential formulation ([1], [3]). A cantilever prismatic beam is hereinafter considered, having length $L$, uniform cross-section of arbitrary shape with area $\Omega$ and contour $\Gamma$. The beam is referred to a counter-clockwise coordinate system with $x$ and $y$ axes coincident with the 
cross-section principal axes of inertia, and $z$ axis orthogonal to the cross-section plane (Figure 1). It is assumed that the beam is subjected to external shear forces $T_{x}$ and $T_{y}$ acting at its terminal section, as well as an external torque $M_{z}$. A complete characterization of the beam stress field is achieved once the normal stresses $\sigma_{z}(z)$ and shear stresses $\tau_{z x}(x, y)$ and $\tau_{z y}(x, y)$ are determined. The normal stresses are directly related to the bending moment induced by the external shear forces:

$$
\sigma_{z}(z)=-\frac{T_{y}(L-z)}{I_{x}} y-\frac{T_{x}(L-z)}{I_{y}} x
$$

where $I_{x}$ and $I_{y}$ are the cross-section inertia moments with respect to the $x$ and $y$ axes, respectively.

For the determination of the shear stresses the equilibrium and compatibility equations have to be considered, along with the boundary and static equivalence conditions. The equilibrium equations can be written in terms of divergence of the shear stress vector field as follows:

$$
\operatorname{div} \boldsymbol{\tau}=\tau_{z x, x}+\tau_{z y, y}=-\sigma_{z, z}=-\frac{T_{y}}{I_{x}} y-\frac{T_{x}}{I_{y}} x \quad \text { in } \Omega
$$

where $\boldsymbol{\tau}^{\mathrm{T}}=\left[\begin{array}{ll}\tau_{z x} & \tau_{z y}\end{array}\right]$, and $f_{, x}$ indicates partial derivative of the function $f$ with respect to the variable $x$. The compatibility conditions are expressed by Beltrami equations:

$$
(1+v) \nabla^{2} \tau_{z x}+J_{1, x z}=0 ; \quad(1+v) \nabla^{2} \tau_{z y}+J_{1, y z}=0 \quad \text { in } \Omega
$$

where $v$ is the Poisson coefficient, $\nabla^{2}(\cdot)=(\cdot)_{, x x}+(\cdot)_{, y y}$ is the Laplace operator, and $J_{1}=\sigma_{x}+\sigma_{y}+\sigma_{z}$ is the first invariant of the stress tensor. By considering eq. (1), Beltrami equations can be expressed in terms of shear stresses laplacian as follows:

$$
\nabla^{2} \tau_{z x}=-\frac{1}{1+v} \frac{T_{x}}{I_{y}} ; \quad \nabla^{2} \tau_{z y}=-\frac{1}{1+v} \frac{T_{y}}{I_{x}} ; \quad \text { in } \Omega
$$


Eqs. (2) and (4) are valid on the whole domain, and they can be combined to obtain the curl of the shear stress vector field:

$$
\mathbf{i}_{z}^{\mathrm{T}} \cdot(\nabla \times \boldsymbol{\tau})=\tau_{z y, x}-\tau_{z x, y}=\frac{v}{1+v}\left(\frac{T_{y} x}{I_{x}}-\frac{T_{x} y}{I_{y}}\right)+2 G \theta
$$

where $\mathbf{i}_{z}$ is the unitary vector in the $z$ axis direction, $G$ is the shear modulus and $\theta$ is the twist rotation per unitary length.

Along the contour $\Gamma$, the free stress boundary conditions have to be fulfilled, that is the shear stress components normal to the contour $\Gamma$ vanish:

$$
\boldsymbol{\tau}^{\mathrm{T}} \mathbf{n}=\tau_{z x} n_{x}+\tau_{z y} n_{y}=0 \quad \text { on } \Gamma
$$

where $\mathbf{n}^{\mathrm{T}}=\left[\begin{array}{ll}n_{x} & n_{y}\end{array}\right]$ is the outward unitary vector normal to the contour $\Gamma$. The flexuretorsion problem is fully characterized once the equivalence conditions between external acting forces and internal shear stresses are considered, that is:

$$
\int_{\Omega} \tau_{z x} d \Omega=T_{x} ; \quad \int_{\Omega} \tau_{z y} d \Omega=T_{y} ; \quad \int_{\Omega}\left(\tau_{z y} x-\tau_{z x} y\right) d \Omega=M_{z}
$$

\section{Formulation by modified complex potential function}

When the cross-section is subjected to pure torsion, the hydrodynamic analogies between the torsion induced shear stress field and the velocity field of a stationary ideal fluid circulating with uniform velocity in a tube of the same cross-section of the twisted beam are well-known ([1]). The formulation of the problem in terms of complex potential function and the definition of the torsion problem in terms of the so-called Prandtl function (corresponding to the stream function of the ideal fluid) result particularly convenient and they allow analytical and series solutions to be determined for some specific cross-sectional geometries ([2], [3], [11]). Herein, a similar approach is proposed for the evaluation of the flexure-torsion problem by defining a modified complex potential function, taking into account the simultaneous 
presence of torque and shear forces. Firstly, a new fictitious stress vector field $\tilde{\boldsymbol{\tau}}=\left[\begin{array}{ll}\tilde{\tau}_{z x} & \tilde{\tau}_{z y}\end{array}\right]$ is introduced as follows:

$$
\tilde{\boldsymbol{\tau}}(x, y)=\boldsymbol{\tau}(x, y)+\mathbf{q}(x, y)+G \theta \mathbf{r} \quad \text { with } \mathbf{q}(x, y)=\left[\begin{array}{l}
q_{x} \\
q_{y}
\end{array}\right]=\frac{1}{m} \mathbf{A I}_{\mathbf{n}} \mathbf{T}
$$

where the vector $\mathbf{T}^{\mathrm{T}}=\left[\begin{array}{ll}T_{x} & T_{y}\end{array}\right]$ have components equal to the acting shear forces, while the vector $\mathbf{r}$, the coefficient $m$, and the matrices $\mathbf{A}$ and $\mathbf{I}_{\mathbf{n}}$ are defined as:

$$
\mathbf{r}=\left[\begin{array}{c}
y \\
-x
\end{array}\right] ; \quad m=2(1+v) I_{x} I_{y} ; \quad \mathbf{A}=\left[\begin{array}{cc}
x^{2} & 2 v x y \\
2 v x y & y^{2}
\end{array}\right] ; \quad \mathbf{I}_{\mathbf{n}}=\left[\begin{array}{cc}
I_{x} & 0 \\
0 & I_{y}
\end{array}\right]
$$

If the stress vector field $\tau$ is solution of the flexure-torsion problem, then the equilibrium and compatibility equations, eqs. (2) and (5) respectively, have to be satisfied. Hence, from the equilibrium equations, it follows:

$$
\operatorname{div} \tilde{\boldsymbol{\tau}}=\operatorname{div} \boldsymbol{\tau}+\frac{T_{y}}{I_{x}} y+\frac{T_{x}}{I_{y}} x=0
$$

while, considering the expression of the curl of $\boldsymbol{\tau}$ eq. (5):

$$
\mathbf{i}_{z}^{\mathrm{T}} \cdot(\nabla \times \tilde{\boldsymbol{\tau}})=\mathbf{i}_{z}^{\mathrm{T}} \cdot(\nabla \times \boldsymbol{\tau})-\frac{v}{1+v}\left(\frac{T_{y} x}{I_{x}}-\frac{T_{x} y}{I_{y}}\right)-2 G \theta=0
$$

Therefore, analogously to the pure torsion problem, it is possible to consider a hydrodynamic analogy between the modified vector field $\tilde{\boldsymbol{\tau}}$ and the velocity field of an incompressible $(\operatorname{div} \tilde{\boldsymbol{\tau}}=0)$ and irrotational $(\nabla \times \tilde{\boldsymbol{\tau}}=0)$ fluid and to define the following complex potential function:

$$
U(w)=\Phi(w)+i \Psi(w)
$$


In eq. (12), $i$ is the imaginary unit, $w=x+i y$ is the complex variable, and the functions $\Phi(w)$ and $\Psi(w)$ are harmonic conjugates, that is they satisfy the following relations:

$$
\begin{array}{ll}
\nabla^{2} \Phi=0 ; & \nabla^{2} \Psi=0 \\
\Psi_{, x}=-\Phi_{, y} ; & \Psi_{, y}=\Phi_{, x} \quad \text { (Cauchy-Riemann equations) }
\end{array}
$$

The function $\Phi$ represents the potential function of the vector field $\tilde{\boldsymbol{\tau}}$ (i.e. $\nabla \Phi=\tilde{\boldsymbol{\tau}}$ ), while $\Psi$ is its stream function. Once the vector field $\tilde{\boldsymbol{\tau}}$ is determined, by definition it is assured that equilibrium and compatibility equations are satisfied. In the following, the free-stress boundary conditions and static equivalence conditions are expressed in terms of the complex potential function.

\section{Free stress boundary conditions}

By substituting the definition of the vector field $\tilde{\boldsymbol{\tau}}$ into eq. (6), the following condition on the boundary is obtained:

$$
(\tilde{\boldsymbol{\tau}}-\mathbf{q}-G \theta \mathbf{r})^{\mathrm{T}} \mathbf{n}=0 \quad \text { on } \Gamma
$$

Eq. (14) can be rewritten in terms of the gradient of the potential function $\Phi$ as follows:

$$
(\nabla \Phi)^{\mathrm{T}} \mathbf{n}=\mathbf{q}^{\mathrm{T}} \mathbf{n}+G \theta \mathbf{r}^{\mathrm{T}} \mathbf{n} \quad \text { on } \Gamma
$$

Considering the $90^{\circ}$ counter-clockwise rotation matrix $\mathbf{R}$ :

$$
\mathbf{R}=\left[\begin{array}{cc}
0 & -1 \\
1 & 0
\end{array}\right]
$$

recalling that $\mathbf{R}^{-1}=\mathbf{R}^{\mathrm{T}}, \mathbf{s}=\mathbf{R} \mathbf{n}$ is the unitary vector tangent to the contour $\Gamma$, and taking advantage of the Cauchy-Riemann relations, eq. (13)b, it follows:

$$
(\nabla \Psi)^{\mathrm{T}} \mathbf{s}=(\mathbf{R} \nabla \Phi)^{\mathrm{T}} \mathbf{s}=(\nabla \Phi)^{\mathrm{T}} \mathbf{R}^{\mathrm{T}} \mathbf{S}=(\nabla \Phi)^{\mathrm{T}} \mathbf{n}
$$


Therefore, the free-boundary stress conditions can be written in terms of stream functions as:

$$
(\nabla \Psi)^{\mathrm{T}} \mathbf{s}=\mathbf{q}^{\mathrm{T}} \mathbf{n}+G \theta \mathbf{r}^{\mathrm{T}} \mathbf{n}=(\mathbf{R} \mathbf{q})^{\mathrm{T}} \mathbf{s}+G \theta(\mathbf{R r})^{\mathrm{T}} \mathbf{s} \quad \text { on } \Gamma
$$

Introducing the function $\Psi_{\theta}(w)$, such that $\nabla \Psi_{\theta}=\mathbf{R r}$, that is:

$$
\Psi_{\theta}=\frac{|w|^{2}}{2}=\frac{x^{2}+y^{2}}{2}
$$

eq. (18) can be rewritten as:

$$
(\nabla \Psi)^{\mathrm{T}} \mathbf{s}-G \theta \nabla \Psi_{\theta} \mathbf{s}=\Psi_{, s}-G \theta \Psi_{\theta, s}=(\mathbf{R q})^{\mathrm{T}} \mathbf{s} \quad \text { on } \Gamma
$$

The contour $\Gamma$ can be generally described by a parametric equation $\xi(t)=(x(t), y(t))$, such that $0 \leq t \leq t_{f}$ and $\xi(0) \equiv \xi\left(t_{f}\right)$. Performing line integration along the contour, the following equation is obtained:

$$
\Psi(t)-G \theta \Psi_{\theta}(t)=b(t)+k \quad \text { on } \Gamma
$$

where $b(t)=\int_{0}^{t}(\mathbf{R q}(t))^{\mathrm{T}} \mathbf{s}(t) d t$ is defined on the boundary and $k=\Psi(0)-G \theta \Psi_{\theta}(0)$ is constant. It is worth to stress that, since the objective is to determine the vector field $\tilde{\boldsymbol{\tau}}$, the stream function $\Psi$ (as well as the potential function $\Phi$ ) can be defined up to a constant without loss of generality. Therefore, in the following, it will be set $k=0$. The function $b(t)$ can be evaluated in exact form for several profiles with known parametric representations, including any polygonal contour, as shown in the numerical application section.

\section{Static equivalence conditions}

As previously mentioned, one of the advantages of the proposed method is that the solution obtained by complex potential automatically satisfies the static equivalence conditions with respect to translation in the $x$ and $y$ directions (Appendix A). Moreover, in this section it is shown how the static equivalence condition with respect to rotation on the cross-section plane 
can be written in terms of potential function $\Phi$. To allow the application of pure boundary methods, the equation is written so that only line integrals along the boundaries are needed.

First, the static equivalence condition eq. (7)c, is rewritten in terms of the vector field $\tilde{\boldsymbol{\tau}}$ and of its potential $\Phi$ :

$$
M_{z}=-\int_{\Omega} \boldsymbol{\tau}^{\mathrm{T}} \mathbf{r} d \Omega=-\int_{\Omega}(\nabla \Phi-\mathbf{q}-G \theta \mathbf{r})^{\mathrm{T}} \mathbf{r} d \Omega
$$

The first term on the right hand side can be written as a line integral along the domain contour by using Stokes' theorem:

$$
\int_{\Omega}(\nabla \Phi)^{\mathrm{T}} \mathbf{r} d \Omega=\int_{\Omega}\left(y \Phi_{, x}-x \Phi_{, y}\right) d \Omega=\int_{\Omega} \nabla \times(\Phi \mathbf{R r}) d \Omega=\oint_{\Gamma} \Phi(t)(\mathbf{R r}(t))^{\mathrm{T}} \mathbf{s}(t) d t(23)
$$

where $\mathbf{s}$ is the unitary vector tangent to the contour. Expanding the second term, the following equation is obtained:

$$
\begin{aligned}
\int_{\Omega} \mathbf{q}^{\mathrm{T}} \mathbf{r} d \Omega & =\frac{1}{m} \int_{\Omega}\left(\left(x^{2} T_{x} I_{x}+2 v x y T_{y} I_{y}\right) y-\left(y^{2} T_{y} I_{y}+2 v x y T_{x} I_{x}\right) x\right) d \Omega= \\
& =\frac{1-2 v}{m}\left(T_{x} I_{x} I_{x y y}-T_{y} I_{y} I_{x x y}\right)
\end{aligned}
$$

where $I_{x y y}=\int_{\Omega} x^{2} y d \Omega$ and $I_{x x y}=\int_{\Omega} y^{2} x d \Omega$ can be calculated as line integral, as well as the other inertia moment, as indicated in Appendix B [16].

Finally, the last term on the right hand side of eq. (22) is:

$$
G \theta \int_{\Omega} \mathbf{r}^{\mathrm{T}} \mathbf{r} d \Omega=G \theta \int_{\Omega}\left(x^{2}+y^{2}\right) d \Omega=G \theta I_{p o l}
$$

where $I_{p o l}$ is the polar inertia moment of the beam cross-section. Hence, the static equivalence condition with respect to the rotation on the cross-section plane can be written as: 


$$
\oint_{\Gamma} \Phi(\mathbf{R r})^{\mathrm{T}} \mathbf{s} d t-G \theta I_{p o l}=-M_{z}+\frac{1-2 v}{m}\left(T_{x} I_{x} I_{x y y}-T_{y} I_{y} I_{x x y}\right)
$$

\section{Shear stress distribution by complex analysis}

The flexure-torsion problem is, now, completely defined, in terms of real and imaginary parts of the complex potential $U$ and $\theta$, by the following set of equations:

$$
\left\{\begin{array}{lc}
\nabla^{2} \Psi=0 & \text { on } \Omega \\
\Psi(t)-G \theta \Psi_{\theta}(t)=b(t) & \text { on } \Gamma \\
\oint_{\Gamma} \Phi(\mathbf{R r})^{\mathrm{T}} \mathbf{s} d t-G \theta I_{p o l}=-M_{z}+\frac{1-2 v}{m}\left(T_{x} I_{x} I_{x y y}-T_{y} I_{y} I_{x x y}\right)
\end{array}\right.
$$

Differently from the classic Dirichlet boundary condition, the value of the stream function on the boundary is herein related to the unknown $\theta$. However, an additional equation is provided by the static equivalence condition with respect to the rotation on the cross-section plane. Since only line integrals need to be evaluated and only boundary conditions are provided, boundary methods involving complex analysis are particularly appropriate for the solution of the mathematical problem described by the set of eqs. (27).

In the following, the Complex Variable Boundary Element Method (CVBEM) has been considered for the numerical implementation. The CVBEM is a boundary method initially developed for Dirichlet-type problems ([21]) and subsequently extended to Neumann problem and mixed boundary condition problems ([22]). It is derived by the Cauchy integral formula, linking the value of analytic function inside a domain with its value on the boundary:

$$
f(w)=\frac{1}{2 \pi i} \oint_{\Gamma} \frac{f(t)}{t-w} d t \quad w \in \Omega
$$

Briefly, by using the CVBEM, the harmonic solution is in general approximated with a function defined in the class [12]: 


$$
f(w)=c_{0}+c_{1} w+\sum_{k=1}^{N} a_{k}\left(w-w_{k}\right) \log _{w_{k}}\left(w-w_{k}\right)
$$

where $c_{0}, c_{1}, a_{1}, \ldots, a_{N}$ are complex coefficients and $w_{1}, \ldots, w_{N}$ are selected nodes on the contour $\Gamma$. The subscript $w_{k}$ in the complex logarithm in eq. (29) indicates that the logarithm should be evaluated with respect to opportunely chosen branch cuts. The latter have to be arbitrary non self-intersecting curves external to the domain $\Omega$ or its boundary $\Gamma$, with exception of the point $w_{k}$, and joining the point $w_{k}$ itself to infinity. This condition is necessary so that the complex logarithm (and, therefore, the function $f(w)$ ) is analytic in the whole domain [12]. In a more recent work ([22]), Whitley and Hromadka suggest to use a broader class of CVBEM functions when an accurate approximation is needed for both the potential function and its gradient. Therefore, in this paper, the following CVBEM functions have been used:

$$
F(w)=c_{0}+c_{1} w+c_{2} \frac{w^{2}}{2}+\sum_{k=1}^{N} a_{k} \frac{1}{2}\left(w-w_{k}\right)^{2}\left(\log _{w_{k}}\left(w-w_{k}\right)-\frac{1}{2}\right)
$$

It is worth to notice that the function $f(w)$ is the derivative of the function $F(w)$. The unknown complex coefficients $c_{0}, c_{1}, c_{2}, a_{1}, \ldots, a_{N}$ can be evaluated by imposing the boundary conditions on selected collocation points.

The CVBEM has been successfully applied to several engineering problems, and in particular, to pure torsion problems of isotropic ([12], [17]) and orthotropic beams ([13]) and to flexure-torsion problems for isotropic beams in absence of boundary singularity points ([18]). In the next section, the proposed complex potential is used for the solution of the flexure-torsion problem of three different cross-sections.

\section{Numerical applications}

By applying the CVBEM to the complex potential $U(w)$ defined in eq. (12), the potential function $\Phi$ and stream function $\Psi$ can be written in the following form: 


$$
\begin{aligned}
& \Phi(x, y)=\gamma_{1} x-\delta_{1} y+\gamma_{2} \frac{x^{2}-y^{2}}{2}-\delta_{2} x y+\sum_{k=1}^{N}\left(\alpha_{k} R_{k}(x, y)-\beta_{k} I_{k}(x, y)\right) \\
& \Psi(x, y)=\gamma_{1} y+\delta_{1} x+\gamma_{2} x y+\delta_{2} \frac{x^{2}-y^{2}}{2}+\sum_{k=1}^{N}\left(\alpha_{k} I_{k}(x, y)+\beta_{k} R_{k}(x, y)\right)
\end{aligned}
$$

where $a_{k}=\alpha_{k}+i \beta_{k}$ and $c_{k}=\gamma_{k}+i \delta_{k}$, while $w_{k}=x_{k}+i y_{k}$ are the selected nodes on the boundary. The functions $R_{k}$ and $I_{k}$ are defined as follows:

$$
\begin{aligned}
& R_{k}(x, y)=\operatorname{Re}\left[\frac{1}{2}\left(w-w_{k}\right)^{2}\left(\log _{w_{k}}\left(w-w_{k}\right)-\frac{1}{2}\right)\right] \\
& I_{k}(x, y)=\operatorname{Im}\left[\frac{1}{2}\left(w-w_{k}\right)^{2}\left(\log _{w_{k}}\left(w-w_{k}\right)-\frac{1}{2}\right)\right]
\end{aligned}
$$

It is worth stressing that, in eqs. (31), the constant $c_{0}$ has been neglected since both functions $\Phi$ and $\Psi$ can be defined up to a constant.

The functions expressed by eqs. (31) satisfy the Laplace equation. The boundary conditions and the static equivalence condition, eqs. (27)b-c, have been used for the determination of the $2 N+4$ unknown coefficients $\left(\gamma_{1}, \gamma_{2}, \alpha_{1}, \ldots, \alpha_{N}\right.$ and $\left.\delta_{1}, \delta_{2}, \beta_{1}, \ldots, \beta_{N}\right)$ and the twist rotation for unitary length $\theta$. First, the contour $\Gamma$ is represented through a parametric equation $\xi(t)=(x(t), y(t))$, with $0 \leq t \leq t_{f}$ and $\xi(0) \equiv \xi\left(t_{f}\right)$. Then, a set of $M$ points $\xi\left(t_{1}\right), \ldots, \xi\left(t_{M}\right)$ are chosen on the boundary; in the following, the points have been selected at regular intervals, however different point distributions can be used. Substituting eq. (31)b into eq. (27)b, the following relation can be written for each point $\xi\left(t_{j}\right)=\left(x\left(t_{j}\right), y\left(t_{j}\right)\right)$ of the contour:

$$
\begin{aligned}
& \gamma_{1} y_{j}+\delta_{1} x_{j}+\gamma_{2} x_{j} y_{j}+\delta_{2} \frac{x_{j}^{2}-y_{j}^{2}}{2}+\sum_{k=1}^{N}\left(\alpha_{k} I_{k}\left(x_{j}, y_{j}\right)+\beta_{k} R_{k}\left(x_{j}, y_{j}\right)\right)-G \theta \frac{x_{j}^{2}+y_{j}^{2}}{2}= \\
& =b\left(x\left(t_{j}\right), y\left(t_{j}\right)\right)
\end{aligned}
$$


providing a set of $M$ different linear algebraic equations in the unknown $\gamma_{1}, \gamma_{2}, \alpha_{1}, \ldots, \alpha_{N}$, $\delta_{1}, \delta_{2}, \beta_{1}, \ldots, \beta_{N}$ and $\theta$. Additionally, by substituting the eq. (31)a into the static equivalence condition eq. (27)c, the following algebraic equation is obtained:

$$
\gamma_{1} J_{x}-\delta_{1} J_{y}+\gamma_{2} J_{x^{2} y^{2}}-\delta_{2} J_{x y}+\sum_{k=1}^{N}\left(\alpha_{k} J_{R k}-\beta_{k} J_{I k}\right)-G \theta I_{p o l}=-M_{z}+\frac{1-2 v}{m}\left(T_{x} I_{x} I_{x y y}-T_{y} I_{y} I_{x x y}\right)
$$

where the following positions have been made:

$$
\begin{array}{ll}
J_{x}=\oint_{\Gamma} x(\mathbf{R r})^{\mathrm{T}} \mathbf{s} d t ; & J_{y}=\oint_{\Gamma} y(\mathbf{R r})^{\mathrm{T}} \mathbf{s} d t ; \\
J_{x^{2} y^{2}}=\frac{1}{2} \oint_{\Gamma}\left(x^{2}-y^{2}\right)(\mathbf{R r})^{\mathrm{T}} \mathbf{s} d t ; & J_{x y}=\oint_{\Gamma} x y(\mathbf{R r})^{\mathrm{T}} \mathbf{s} d t ; \\
J_{R k}=\oint_{\Gamma} R_{k}(\mathbf{R r})^{\mathrm{T}} \mathbf{s} d t ; & J_{I k}=\oint_{\Gamma} I_{k}(\mathbf{R r})^{\mathrm{T}} \mathbf{s} d t ; \quad k=1, \ldots, N
\end{array}
$$

The solution of the flexure-torsion problem is, then, determined by a set of $M+1$ linear algebraic equations in the $2 N+5$ unknowns $\gamma_{1}, \gamma_{2}, \alpha_{0}, \ldots, \alpha_{N}, \delta_{1}, \delta_{2}, \beta_{0}, \ldots, \beta_{N}$ and $\theta$. The minimum number of collocation points to achieve the solution of the problem is, obviously, $M=2 N+4$; however, selecting a larger number of points and solving the subsequent overdetermined system of equations by the pseudo-inverse matrix method lead to increased convergence of the procedure. For such reason, in the following applications, $M=2(2 N+5)$ has been used, that is twice the number of the unknowns. It is worth notice that the function $b(t)$ is only dependent on the geometry of the cross-section and not on the selected boundary nodes or collocation points, and, therefore, it has to be evaluated only once for any degree of discretization of the boundary.

Three numerical applications are proposed for the three domains illustrated in Figure 2. The algorithm for the determination of the shear stress distributions and the twist rotation for unitary length can be summarized by the following steps:

- definition of the parametric equation $\xi(t)=(x(t), y(t))$ representing the contour $\Gamma$ of the analyzed domain; $\xi(t)$ can either be a continuous (e.g., ellipse) or a piecewise 
function (e.g., L-shaped cross-section); the definition of the cross-section geometrical properties follows straightforwardly, as shown in Appendix A;

- selection of the $N$ nodes on the boundary and the related branch-cuts;

- selection of the $M=2(2 N+5)$ collocation points on the boundary; in the following, both the nodes and the collocation points are uniformly distributed along the contour of the cross-section;

- definition of the linear algebraic system of equations $\mathbf{C X}=\mathbf{B}$, where $\mathbf{C}$ is the $M \times(2 N+5)$ matrix of the coefficients of eqs. (33) and (34), $\mathbf{X}$ is the array containing the $2 N+5$ unknowns $\gamma_{1}, \gamma_{2}, \alpha_{0}, \ldots, \alpha_{N}, \delta_{1}, \delta_{2}, \beta_{0}, \ldots, \beta_{N}$ and $\theta$, and $\mathbf{B}$ is the array having as elements the right hand side terms of eqs. (33) and (34);

- solution of the system $\mathbf{C X}=\mathbf{B}$ by pseudo-inverse matrix method.

Once the unknowns have been determined, the complex potential can be calculated by eqs. (31). The components of the fictitious vector field $\tilde{\boldsymbol{\tau}}$ are obtained as partial derivatives of the potential $\Phi$, taking into account eq. (30):

$$
\begin{aligned}
& \tilde{\tau}_{z x}(x, y)=\gamma_{1}+\gamma_{2} x-\delta_{2} y+\sum_{k=1}^{N}\left(\alpha_{k} r_{k}(x, y)-\beta_{k} i_{k}(x, y)\right) \\
& \tilde{\tau}_{z y}(x, y)=-\left(\delta_{1}+\gamma_{2} y+\delta_{2} x+\sum_{k=1}^{N}\left(\alpha_{k} i_{k}(x, y)+\beta_{k} r_{k}(x, y)\right)\right)
\end{aligned}
$$

where the functions $r_{k}$ and $i_{k}$ are defined as:

$$
\begin{aligned}
& r_{k}(x, y)=\operatorname{Re}\left[\left(w-w_{k}\right)\left(\log _{w_{k}}\left(w-w_{k}\right)\right)\right] \\
& i_{k}(x, y)=\operatorname{Im}\left[\left(w-w_{k}\right)\left(\log _{w_{k}}\left(w-w_{k}\right)\right)\right]
\end{aligned}
$$

Finally, the shear stress vector field $\tau$ is determined by eq. (8) as follows:

$$
\boldsymbol{\tau}(x, y)=\tilde{\boldsymbol{\tau}}(x, y)-\mathbf{q}(x, y)-G \theta \mathbf{r}
$$


The algorithm has been implemented using the computational software Wolfram Mathematica ${ }^{\circledR}$ on a 64-bit, 2.4GHz Intel(R) Core(TM) i7-3630QM CPU, with limited use of parallel processing on the four available cores for the evaluation of the line integrals defined in eq. (35). Details on the results for each studied cross-section, together with the required computational times, are reported in the next sections.

\section{Elliptical cross-section}

The first proposed example is an elliptical cross-section with semi-axes $l=2$ and $h=1$ (Figure 2a), Poisson modulus $v=0.3$ and shear modulus $G=1$. The closed-form solution for the flexure-torsion problem in an elliptical domain is well-known [1], and, therefore, this domain is herein used as a benchmark to test results obtained by the CVBEM. The following parameter equations for the elliptic contour have been considered:

$$
\left\{\begin{array}{l}
x(t)=l \cos (t) \\
y(t)=h \sin (t)
\end{array} \quad 0 \leq t \leq 2 \pi\right.
$$

In this case, the function $b(t)$ on the boundary can be evaluated analytically:

$$
b(t)=\frac{2 T_{x} h \sin (t)\left(3+(2 v-1) \sin ^{2}(t)\right)-2 T_{y} l \cos (t)\left(3+(2 v-1) \cos ^{2}(t)\right)}{3 \ln \pi(1+v)}
$$

The case of shear forces and twist moment acting simultaneously has been considered ( $\left.T_{x}=1 ; T_{y}=1 ; M_{z}=1\right)$. The boundary has been discretized using $N=6$ nodes. The procedure returns $\theta=0.19894$, matching the value of the twist rotation provided by the exact solution. The required computational time has been of 16.00 seconds. Results in terms of shear stresses along the two axes of the ellipse are depicted in Figures 3a-b and compared with the exact solution, showing perfect agreement.

\section{L-shaped cross-section}

When dealing with problems governed by Laplace equation, the L-shaped domain is an extensively studied boundary singularity problem, due to its reentrant corner. Herein, the L- 
shaped cross-section shown in Figure $2 \mathrm{~b}$ has been considered, with $l=1$, Poisson modulus $v=0.3$ and shear modulus $G=1$. The following piecewise parameter equations have been used to represent the boundary of the domain:

$$
\begin{array}{r}
x(t)=\frac{\sqrt{2}}{2} \begin{cases}-t & 0 \leq t<2 \\
t-4 & 2 \leq t<6 \\
-(t-8) & 6 \leq t \leq 8\end{cases} \\
y(t)=\frac{\sqrt{2}}{6} \begin{cases}-(3 t-5) & 0 \leq t<3 \\
3 t-13 & 3 \leq t<4 \\
-(3 t-11) & 4 \leq t<5 \\
3 t-10 & 5 \leq t \leq 8\end{cases}
\end{array}
$$

Performing line integration, the function $b(t)$ has been evaluated analytically, obtaining the piecewise function:

$$
b(t)=\frac{3 T_{x} I_{x} P_{k}(t)+T_{y} I_{y} Q_{k}(t)}{36 \sqrt{2} I_{x} I_{y}(1+v)} \quad t_{k-1} \leq t \leq t_{k} ; \quad k=1, \ldots, 6
$$

where $P_{k}(t)$ and $Q_{k}(t)$ are third order polynomials, reported in details in Appendix C. Three cases have been analyzed: pure torsion $\left(M_{z}=1\right)$ and pure shear forces acting in the $x$ and $y$ directions ( $T_{x}=1$ and $T_{y}=1$, respectively). Shear vector fields and shear stress magnitudes for the L-shaped domain, for the three cases, are depicted in Figures 4a-f. Table 1 reports results in terms of the torsional inertia moment $J_{t}=M_{z} /(G \theta)$ and $y$ coordinate of the shear center $C_{y}$. Results have been evaluated using the herein proposed formulation by CVBEM, for various degrees of boundary discretization. The required computational times varies from 11.12 seconds for 48 nodes to 73.22 seconds in the case of 240 nodes. The determination of the shear center position is straightforward by using Betti's theorem [16]. First, the two cases $M_{z}=1$ and $T_{x}=1$ are solved and the two unitary rotations of the cross-section are obtained, here labeled $\theta_{z}$ and $\theta_{x}$, respectively. Then, following Betti's theorem, $C_{y}=\theta_{x} / \theta_{z}$. 
The same numerical values have been compared with values obtained by FEM, implemented according to [23]-[24], considering several finite element discretization degrees in order to achieve numerical stability, and showing excellent agreement of the results. Moreover, CVBEM using the formulation proposed in [18] has been implemented as well. As shown, for the latter case, the presence of a boundary singularity point greatly affects the method, and the obtained solutions largely diverge from the exact ones.

\section{Epicycloid-shaped cross-section}

In this section, results obtained for an epicycloid-shaped cross-section are reported. Cusps of an epicycloid corresponds to boundary singularities points, similarly to the L-shaped domain. Here a 4 cusps epicycloid is considered (Figure 2c), whose parametric equations are:

$$
\left\{\begin{array}{l}
x(t)=r\left(\cos (t)+\frac{1}{5} \cos (5 t)\right) \\
y(t)=r\left(\sin (t)+\frac{1}{5} \sin (5 t)\right)
\end{array} \quad 0 \leq t \leq 2 \pi\right.
$$

It has been considered $r=1$, Poisson modulus $v=0.3$ and shear modulus $G=1$. Also in this case, the function $b(t)$ can be evaluated in closed form as follows:

$$
b(t)=\frac{1}{3348 a \pi(1+v)} \sum_{n=1}^{8} A_{n}\left(T_{x} \sin ((2 n-1) t)+(-1)^{n} T_{y} \cos ((2 n-1) t)\right) \quad 0 \leq t \leq 2 \pi
$$

where the coefficients $A_{n}$ are reported in details in Appendix C. Values of $J_{t}$ evaluated by the proposed approach for the case of unitary external torsional moment $\left(M_{z}=1\right)$ are reported in Table 2. In the latter, results are compared with those obtained by FEM, also in this case implemented according to [23]-[24]. Figures 5a-b show the shear vector field and shear magnitudes contour plot for the case of pure torsion $\left(M_{z}=1\right)$, while Figures $5 \mathrm{c}-\mathrm{d}$ show results for a unitary external shear force acting along the $y$ axis direction $\left(T_{y}=1\right)$. Due to the symmetry of the studied domain, the case of shear acting along the $x$ axis has not been 
reported. The required computational times varies from 28.73 seconds for 48 nodes to 152.10 seconds in the case of 480 nodes. As it can be noticed, the results are not affected by the presence of multiple boundary singularity points and they converge for increasing degree of boundary discretization.

\section{Conclusions}

In this paper, a novel complex potential formulation is proposed for determining the shear stress distribution, as well as the unitary twist rotation of the cross-section, of De Saint Venant beams subjected to external twist moments and shear forces. The new formulation is based on the definition of a fictitious incompressible irrotational vector field, so that its potential function and stream function are easily determined by hydrodynamic analogy. The flexure-torsion problem is completely defined as a Dirichlet problem in terms of the stream function of the fictitious vector field, coupled with the static equivalence condition with respect to rotation of the cross-section, written in terms of potential function. The use of the proposed complex potential guarantees that the static equivalence conditions with respect to translation is automatically satisfied. All domain integrals are rewritten as line integrals, so that the solution of the method can be achieved by using boundary methods.

The proposed formulation has been validated by opportunely implementing the Complex Variable Boundary Element Method. The solution, obtained for an elliptical crosssection subjected simultaneously to shear and torsion, has been used as benchmark to test the method accuracy. By using CVBEM, very few nodes are needed to achieve high accuracy in the results.

The formulation has been tested for domains with single and multiple boundary singularity points, considering in particular a L-shaped and an epicycloid-shaped crosssections. Adopting the CVBEM, solutions are stable and converge for increasing degree of the boundary discretization. Numerical values have been compared with Finite Element Method analysis, obtaining matching results. With respect to the latter, the use of CVBEM has the great advantage of easiness in the boundary discretization with respect to the meshing process of the FEM. Moreover, while FEM returns values of the shear stresses in selected 
nodes, the CVBEM returns the complete shear stress distribution in all the points in the domain.

The proposed formulation can be implemented by numerical methods different from the CVBEM. Although complex boundary element methods seem to be the most suitable approaches, other boundary methods, as well as finite element methods or mesh-less approaches, can be used as well.

In the case of composite or heterogeneous cross-sections with anisotropic behavior, the presence of the additional elastic constants in the constitutive equations has to be considered. A generalized complex potential approach and its application for the solution of the flexure-torsion problem for cross-sections with boundary singularity points is currently under investigations.

\section{Appendix A: static equivalence conditions with respect to translations}

This appendix shows that the two static equivalence conditions with respect to translations, eqs. (7a-b), are automatically satisfied by the stress vector field obtained through the proposed complex potential. By substituting the expression of the vector field $\tilde{\tau}$ into eq. (7a), and recalling that $\Phi_{, x}=\tau_{z x}$, it follows:

$$
\int_{\Omega} \tau_{z x} d \Omega=\int_{\Omega}\left(\Phi_{, x}-q_{x}-G \theta y\right) d \Omega
$$

Since $\nabla^{2} \Phi=0$, the first term on the right hand side of eq. (A.1) can be rewritten as:

$$
\int_{\Omega} \Phi_{, x} d \Omega=\int_{\Omega}\left(\Phi_{, x}+x \nabla^{2} \Phi\right) d \Omega=\int_{\Omega}\left(\left(\Phi_{, x}+x \Phi_{, x x}\right)+x \Phi_{, y y}\right) d \Omega=\int_{\Omega} \operatorname{div}(x \nabla \Phi) d \Omega
$$

and, by taking advantage of Gauss's theorem:

$$
\int_{\Omega} \Phi_{, x} d \Omega=\oint_{\Gamma} x(\nabla \Phi)^{\mathrm{T}} \mathbf{n} d t
$$


Since the line integral in eq. (A.3) is performed along the contour $\Gamma$, the boundary conditions eq. (15) hold true; therefore the following expression is obtained:

$$
\int_{\Omega} \Phi_{, x} d \Omega=\oint_{\Gamma} x(\mathbf{q}+G \theta \mathbf{r})^{\mathrm{T}} \mathbf{n} d t
$$

and by applying again Gauss’s theorem:

$$
\int_{\Omega} \Phi_{, x} d \Omega=\int_{\Omega} \operatorname{div}(x \mathbf{q}) d \Omega+G \theta \int_{\Omega} \operatorname{div}(x \mathbf{r}) d \Omega=\int_{\Omega}\left(q_{x}+G \theta y+x \operatorname{div}(\mathbf{q}+G \theta \mathbf{r})\right) d \Omega(\text { A.5) }
$$

Substituting eq. (A.5) back into eq. (A.1):

$$
\int_{\Omega} \tau_{z X} d \Omega=\int_{\Omega} x \operatorname{div}(\mathbf{q}+G \theta \mathbf{r}) d \Omega
$$

Finally, by considering the definition of the vector field $\tilde{\tau}$ eq. (8), recalling the expression of the divergence of the stress vector field $\boldsymbol{\tau}$ and recalling that $\operatorname{div} \tilde{\boldsymbol{\tau}}=0$, the following expression is obtained:

$$
\int_{\Omega} \tau_{z x} d \Omega=\int_{\Omega} x \operatorname{div}(\tilde{\boldsymbol{\tau}}-\boldsymbol{\tau}) d \Omega=\frac{T_{y}}{I_{x}} \int_{\Omega} x y d \Omega+\frac{T_{x}}{I_{y}} \int_{\Omega} x^{2} d \Omega
$$

where the integral in the first term is null, since the cross-section is referred with respect to the principal inertia axes, while the second one is the inertia moment $I_{y}$. Therefore, the first static equivalence condition is verified:

$$
\int_{\Omega} \tau_{z x} d \Omega=T_{x}
$$

By following an analogous reasoning, permuting $x$ and $y$, it can be demonstrated that the static equivalence condition with respect to translation in the $y$ direction is verified as well. 


\section{Appendix B: cross-section geometrical properties by line integrals}

For any domain, geometrical properties like area, static and inertia moments can be evaluated by using line integrals, taking advantage of the Green's theorem [16] as follows:

$$
\begin{aligned}
& A=\int_{\Omega} d \Omega=\frac{1}{2} \oint_{\Gamma}\left(x n_{x}+y n_{y}\right) d t \\
& S_{x}=\int_{\Omega} y d \Omega=\frac{1}{2} \oint_{\Gamma} y^{2} n_{y} d t ; \quad S_{y}=\int_{\Omega} x d \Omega=\frac{1}{2} \oint_{\Gamma} x^{2} n_{x} d t \\
& I_{x}=\int_{\Omega} y^{2} d \Omega=\frac{1}{3} \oint_{\Gamma} y^{3} n_{y} d t ; \quad I_{y}=\int_{\Omega} x^{2} d \Omega=\frac{1}{3} \oint_{\Gamma} x^{3} n_{x} d t \\
& I_{x y y}=\int_{\Omega} x^{2} y d \Omega=\frac{1}{3} \oint_{\Gamma} x^{3} y n_{x} d t ; \quad I_{y x x}=\int_{\Omega} y^{2} x d \Omega=\frac{1}{3} \oint_{\Gamma} y^{3} x n_{y} d t
\end{aligned}
$$

\section{Appendix C: closed-form expressions of the boundary function $b(t)$ for the studied domains.}

The boundary function $b(t)$ has been introduced in eq. (21), and it is here reported for sake of clarity:

$$
b(t)=\int_{0}^{t}(\mathbf{R q})^{\mathrm{T}} \mathbf{s} d t=\frac{1}{2 I_{x} I_{y}(1+v)} \int_{0}^{t}\left(\left(I_{x} T_{x} x^{2}+2 v I_{y} T_{y} x y\right) s_{y}-\left(2 v I_{x} T_{x} x y+I_{y} T_{y} y^{2}\right) s_{x}\right) d t
$$

where the dependence on the parameter $t$, along the boundary, of the coordinates $x$ and $y$, as well as the components of the unitary vector $\mathbf{s}$, tangent to the contour, has herein been omitted. The function $b(t)$ can be determined in closed-form for most practical domain, including any polygonal shape, as well as several cross-sections whose boundary is described by continuous or piecewise parametric functions. Herein, the explicit expressions for the Lshaped and epicycloid-shaped cross-sections previously analyzed are reported. 


\section{L-shaped cross-section}

For the L-shaped cross-section depicted in Figure 2b, whose contour is described by the parametric equations (41), the boundary function $b(t)$ assumes the form:

$$
b(t)=\frac{3 T_{x} I_{x} P_{k}(t)+T_{y} I_{y} Q_{k}(t)}{36 \sqrt{2} I_{x} I_{y}(1+v)} \quad t_{k-1} \leq t \leq t_{k} ; \quad k=1, \ldots, 6
$$

where the third order polynomials $P_{k}(t)$ and $Q_{k}(t)$ are expressed as follows:

$$
\begin{aligned}
& P_{1}(t)=t^{2}((2 v-1) t-5 v) \\
& Q_{1}(t)=t\left(-3(2 v-1) t^{2}+15(v-1) t+25\right) \\
& P_{2}(t)=(2 v-1) t^{3}+(12-17 v) t^{2}+(5 v-6) t+16(3-2 v) \\
& Q_{2}(t)=3(2 v-1) t^{3}+3(5-17 v) t^{2}+5(24 v-5) t+4(7-18 v) \\
& P_{3}(t)=-(2 v-1) t^{3}+(25 v-12) t^{2}+8(6-13 v) t+26(5 v-3) \\
& Q_{3}(t)=(t-4)\left(3(2 v-1) t^{2}+3(9-17 v) t+(108 v-61)\right) \\
& P_{4}(t)=(2 v-1) t^{3}+(12-23 v) t^{2}+8(11 v-6) t+2(25-63 v) \\
& Q_{4}(t)=(t-4)\left(3(2 v-1) t^{2}+3(7-15 v) t+(84 v-37)\right) \\
& P_{5}(t)=-(2 v-1) t^{3}+(31 v-12) t^{2}+8(6-19 v) t+(14 v-5) \\
& Q_{5}(t)=3(2 v-1) t^{3}+3(19-31 v) t^{2}+19(24 v-19) t+2(374-348 v) \\
& Q_{6}(t)=(t-8)\left(-3(2 v-1) t^{2}+3 t(27 v-11)+(97-264 v)\right)
\end{aligned}
$$




\section{Epicycloid-shaped cross-section}

For the epicycloid-shaped cross-section depicted in Figure 2c, whose contour is described by the parametric equations (43), the boundary function $b(t)$ assumes the form:

$$
b(t)=\frac{1}{3348 a \pi(1+v)} \sum_{n=1}^{8} A_{n}\left(T_{x} \sin ((2 n-1) t)+(-1)^{n} T_{y} \cos ((2 n-1) t)\right) \quad 0 \leq t \leq 2 \pi
$$

where the coefficients $A_{n}$ are reported in the following:

$$
\begin{array}{ll}
A_{1}=45(97+70 v) ; & A_{2}=900 ; \\
A_{3}=9(73+22 v) ; & A_{4}=225(1-2 v) ; \\
A_{5}=5(11+2 v) ; & A_{6}=45(1-2 v) ; \\
A_{7}=0 ; & A_{8}=3(1-2 v) ;
\end{array}
$$

\section{References}

[1] Timoshenko, S., Goodier, J.N. (1951), “Theory of elasticity”, New York, McGraw-Hill.

[2] Muskhelishvili, N.I. (1953), "Some basic problems of mathematical theory of elasticity”, Groningen: P. Noordhoff Ltd.

[3] Sokolnikoff, I.S. (1956), "Mathematical theory of elasticity”, McGraw-Hill Book Company.

[4] Mason, W.E., Hermann, L.R., "Elastic shear analysis of general prismatic beams", ASCE Proceedings, Journal of the Engineering Mechanics Division, 94(EM4), 965983.

[5] Krahula, J.L., Lauterbach, G.F. (1969), “A finite element solution for Saint-Venant torsion”, AIAA Journal, 7 (12), 2200-2203.

[6] Gruttmann, F., Sauer, R., Wagner, W. (1999), "Shear stresses in prismatic beams with arbitrary cross-sections”, International Journal for Numerical methods in Engineering, 45, 865-889.

[7] Lacarbonara, W., Paolone, A., (2007), "On solution strategies to Saint-Venant problem”, Journal of Computational and Applied Mathematics, 206, 473-497.

[8] Chou, S.I., (1993), "Determination of centers of flexure using the boundary element method”, Engineering Analysis with Boundary Element, 12, 321-324.

[9] Friedman, Z., Kosmatka, J.B., (2000), "Torsion and flexure of a prismatic isotropic beam using the boundary element method", Computers and Structures, 74, 479-494.

[10] Sapountzakis, E.J., Protonotariou, V.M., (2008), “A displacement solution for transverse shear loading of beams using the boundary element method", Computer and Structures, 86, 771-779.

[11] England, A.H., (1971), "Complex variable methods in elasticity”, Wiley-Interscience.

[12] Hromadka II, T.V., Whitley, R.J., (1998), "Advances in the Complex Variable Boundary Element Method”, Springer, New York. 
[13] Dumir, P.C., Kumar, R., (1993), “Complex variable boundary element method for torsion of anisotropic bars”, Applied Mathematical Modelling, 17, 80-88.

[14] Di Paola, M., Pirrotta, A., Santoro, R., (2008), "Line Element-less Method (LEM) for beam torsion solution (truly no-mesh method)”, Acta Mechanica, 195(1-4), 349-364.

[15] Barone, G., Pirrotta, A., Santoro, R., (2011), "Comparison among three boundary element methods for torsion problems: CPM, CVBEM, LEM”, Engineering Analysis with Boundary Elements, 35, 895-907.

[16] Di Paola, M., Pirrotta, A., Santoro, R. (2011), "De Saint Venant flexure-torsion problem handled by Line Elemen-less Method (LEM)”, Acta Mechanica, 217(1-2), 110-118.

[17] Barone, G., Pirrotta, A., (2013), "CVBEM application to a novel potential function providing stress field and twist rotation at once", Journal of Engineering Mechanics, 139(9), 1290-1293.

[18] Barone, G., Pirrotta, A. (2013), “CVBEM for solving De Saint-Venant solid under shear forces”, Engineering Analysis with Boundary Elements, 37(2), 197-204.

[19] Santoro, R. (2010), "The line element-less method analysis of orthotropic beam for the De Saint Venant torsion problem”, International Journal of Mechanical Sciences, 52(1), 43-55.

[20] Santoro, R. (2011), "Solution of De Saint Venant flexure-torsion problem for orthotropic beam via LEM (Line Element-less Method)", European Journal of Mechanics A/Solids, 30(6), 924-939.

[21] Hromadka II, T.V., Lai, C, (1987), "The complex boundary element method in engineering analysis”, Springer, New York.

[22] Whitley, R.J., Hromadka II, T.V., (2006), “Theoretical developments in the complex variable boundary element method”, Engineering Analysis with Boundary Elements, 30, 1020-1024.

[23] Gruttmann, F. e Wagner, W. (2001), "Shear correction factors in Timoshenko's beam theory for arbitrary shaped cross-sections”, Computational Mechanics, vol. 27(3), p. 199-207.

[24] Zienkiewicz OC, Taylor RL (1989), “The Finite Element Method”, McGraw-Hill, London. 


\section{Table 1}

Torsional inertia moment $J_{t}$ and $y$ coordinate of the shear center $C$ for L-shaped cross-section.

\begin{tabular}{ccccccccc}
\hline & CVBEM & \multicolumn{3}{c}{ FEM } & \multicolumn{3}{c}{ CVBEM $^{(*)}$} \\
\hline Nodes & $J_{t}$ & $C_{y}$ & Nodes & $J_{t}$ & $C_{y}$ & Nodes & $J_{t}$ & $C_{y}$ \\
48 & 0.8607 & 0.2643 & 451 & 0.8595 & 0.2614 & 48 & 1.5292 & -0.2646 \\
96 & 0.8580 & 0.2665 & 2383 & 0.8572 & 0.2627 & 96 & 1.8034 & -0.4814 \\
144 & 0.8573 & 0.2670 & 8259 & 0.8566 & 0.2632 & 144 & 2.0491 & -0.6757 \\
192 & 0.8570 & 0.2673 & 30063 & 0.8564 & 0.2633 & 192 & 2.2754 & -0.8547 \\
240 & 0.8568 & 0.2674 & 44283 & 0.8564 & 0.2634 & 240 & 2.4873 & -1.0223 \\
\hline
\end{tabular}

${ }^{(*)}$ CVBEM considering the formulation based on the shear stresses proposed in [18]. 


\section{Table 2}

Torsional inertia moment $J_{t}$ for epicycloid-shaped cross-section.

\begin{tabular}{cccc}
\hline \multicolumn{2}{c}{ CVBEM } & \multicolumn{2}{c}{ FEM } \\
\hline Nodes & $J_{t}$ & Nodes & $J_{t}$ \\
48 & 1.8503 & 561 & 1.8311 \\
96 & 1.8390 & 1377 & 1.8302 \\
144 & 1.8367 & 11879 & 1.8283 \\
192 & 1.8358 & 32425 & 1.8254 \\
240 & 1.8354 & 53621 & 1.8253 \\
480 & 1.8349 & 74481 & 1.8253 \\
\hline
\end{tabular}




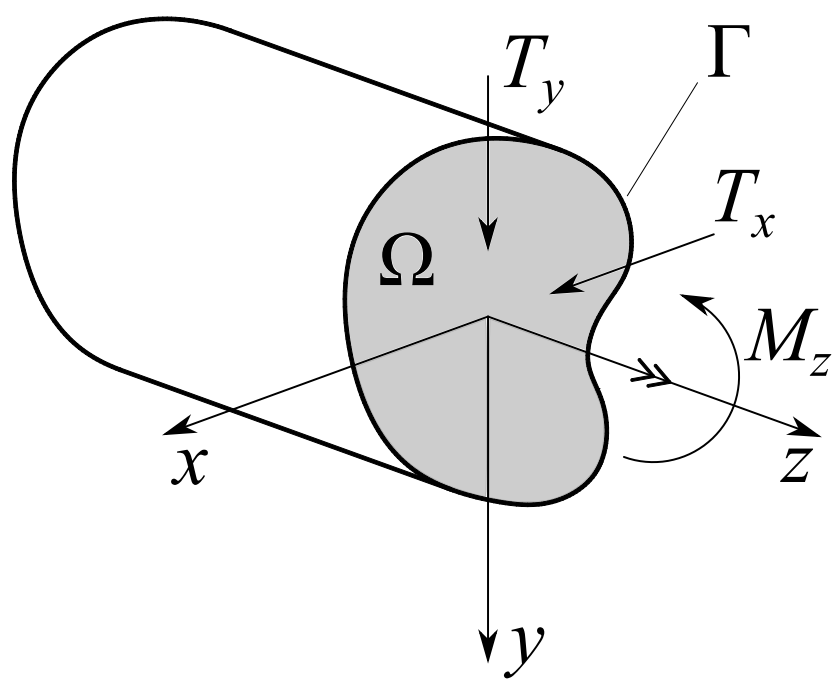

FIGURE 1: De Saint Venant beam subjected to external shear and torsion excitations. 
Figure 2

a)

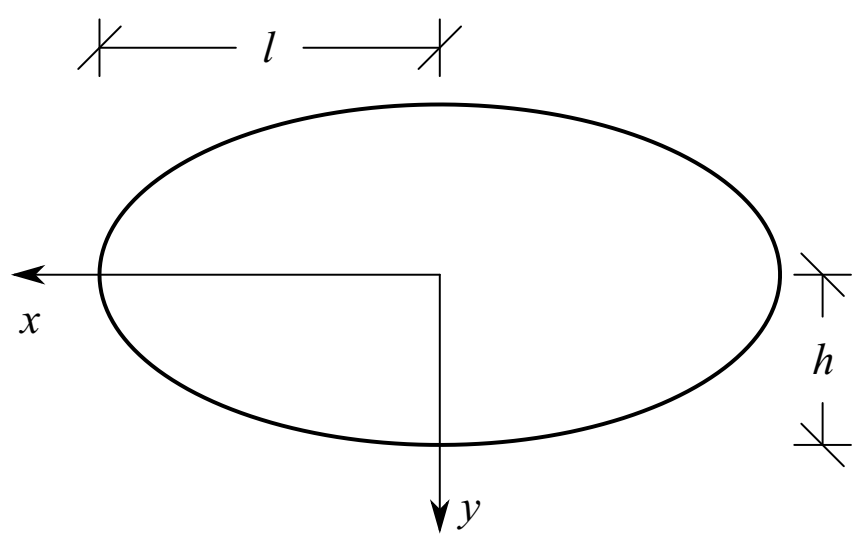

c) $\Varangle 1.2 r \longrightarrow$

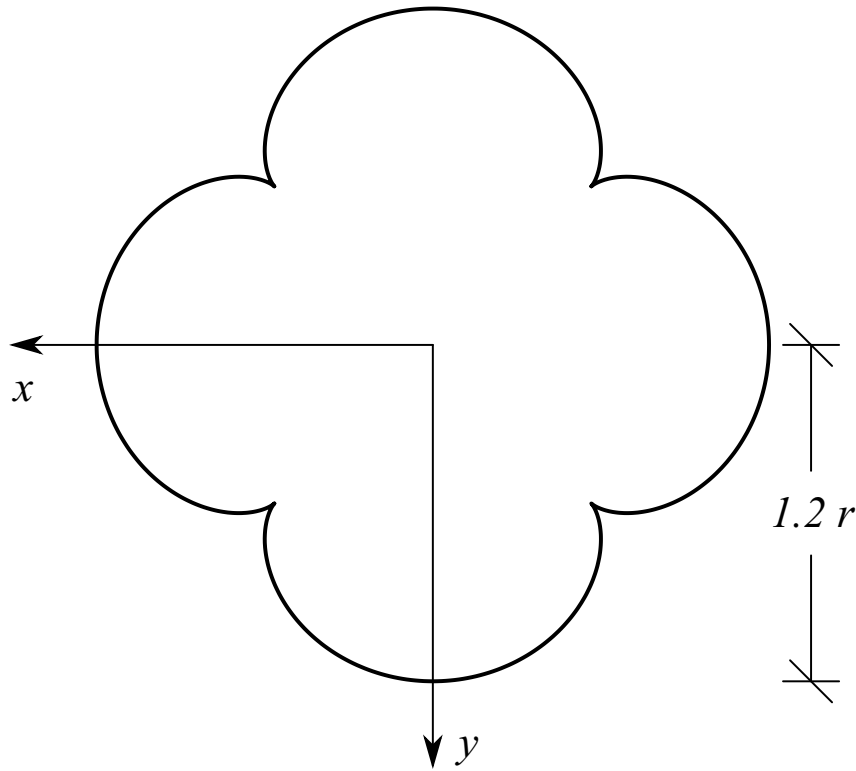

b)

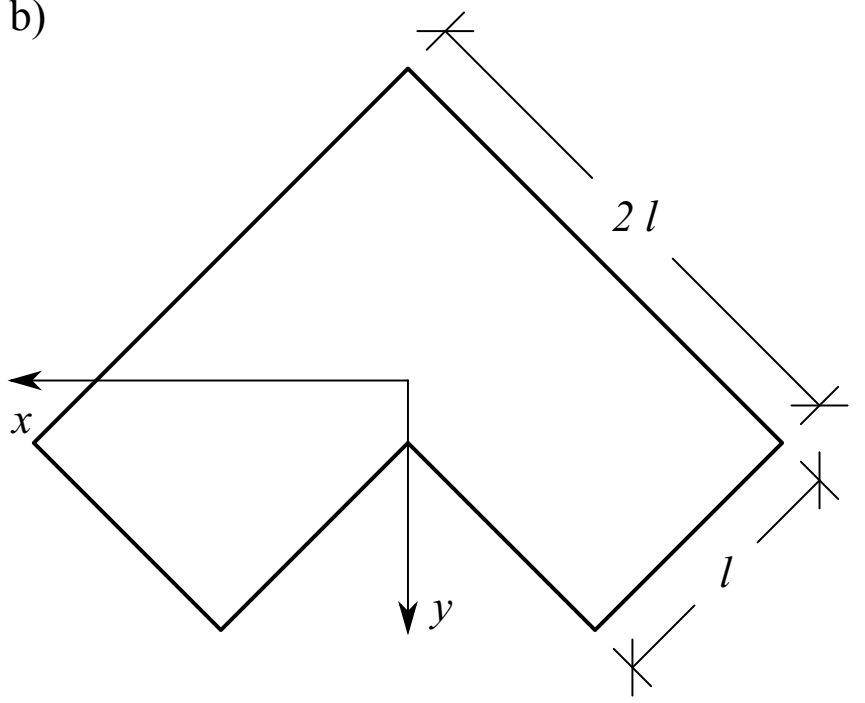

FIGURE 2: Analyzed cross-sectional geometries: (a) elliptic cross-section; (b) L-shaped cross-section; (c) epicycloid-shaped cross-section 
a)

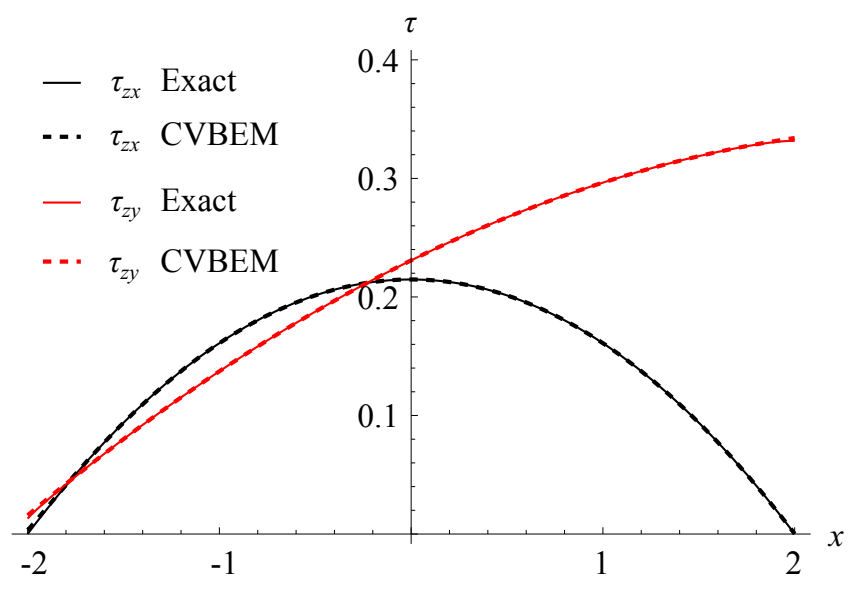

b)

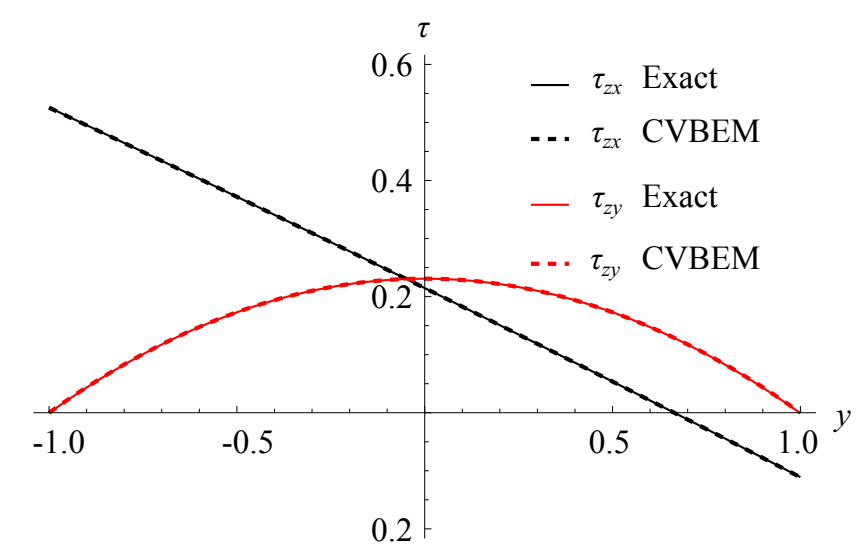

FIGURE 3: Shear stress functions in elliptic cross-section along the (a) $x$ axis and (b) $y$ axis for shear and torsion acting simultaneously $\left(T_{x}=1, T_{y}=1, M_{z}=1\right)$. Exact solution (continuous lines) and CVBEM (dashed lines). 
a)

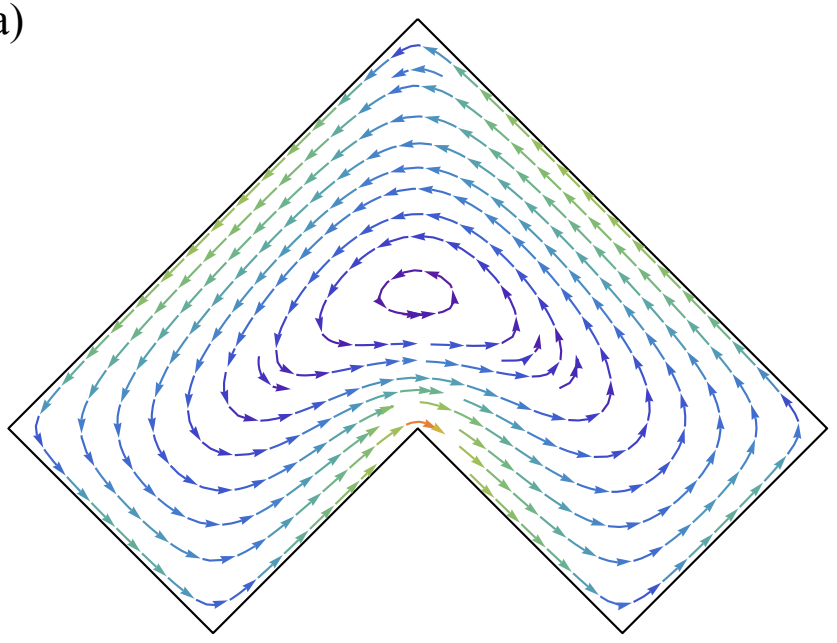

c)

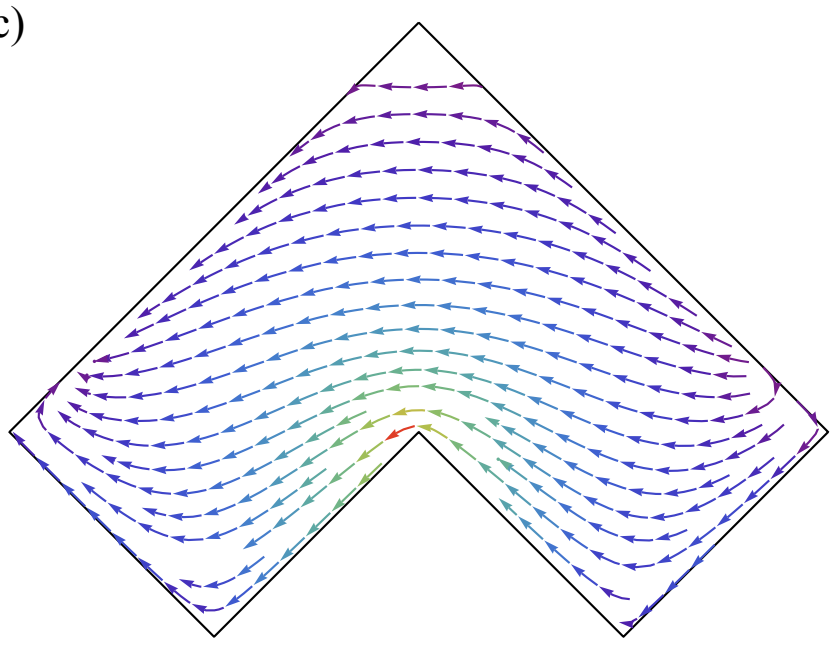

e)

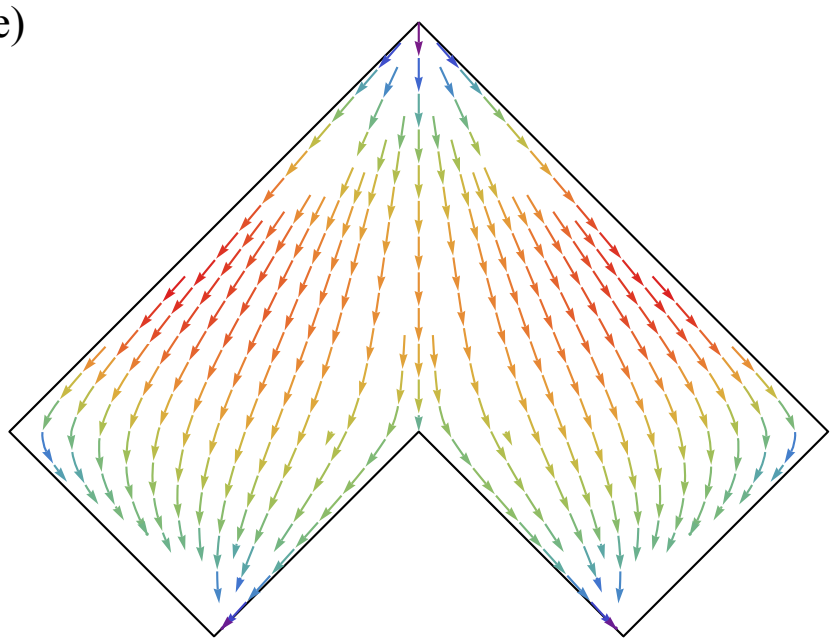

b)

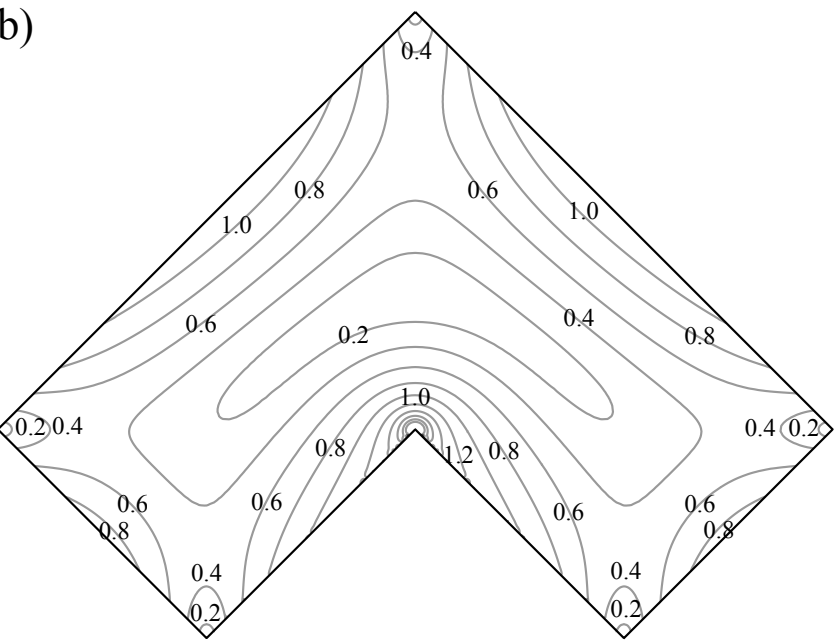

d)

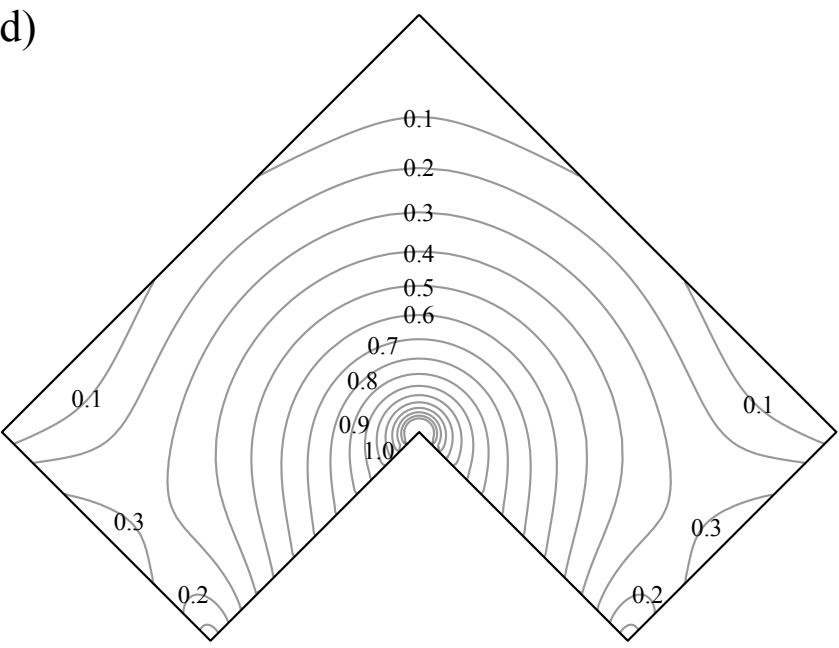

f)

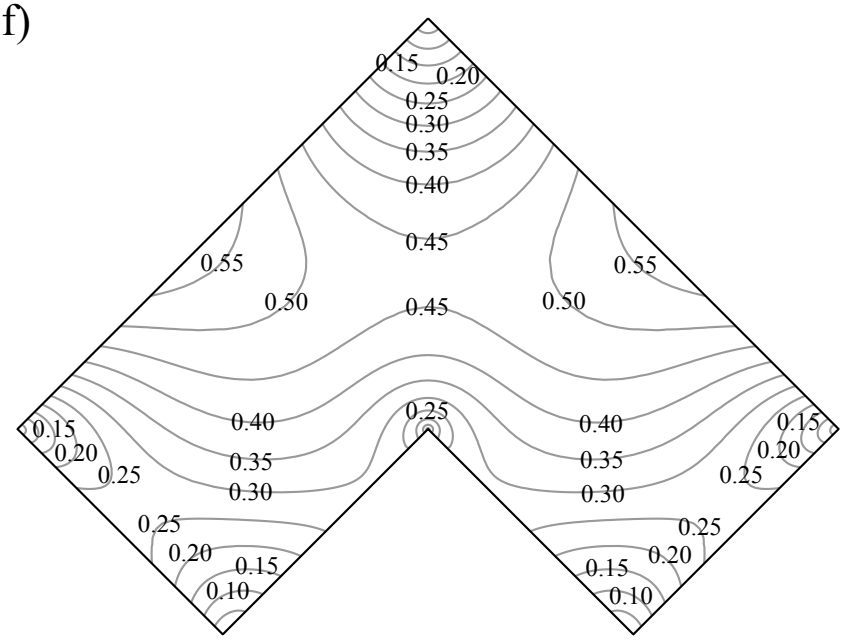

FIGURE 4: Shear stress vector fields and shear stress magnitude contour plots in L-shaped cross-section evaluated by CVBEM considering (a-b) pure torsion $\left(M_{z}=1\right)$; (c-d) shear along the $x$ direction $\left(T_{x}=1\right)$; (e-f) shear along the $y$ direction $\left(T_{y}=1\right)$. 
Figure 5

a)

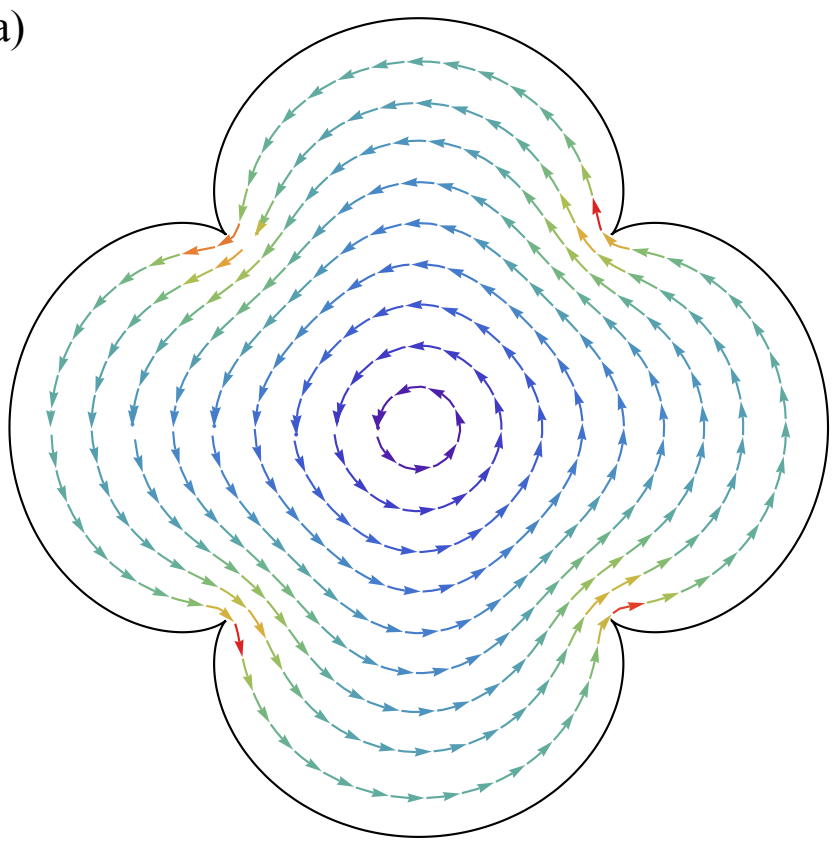

c)

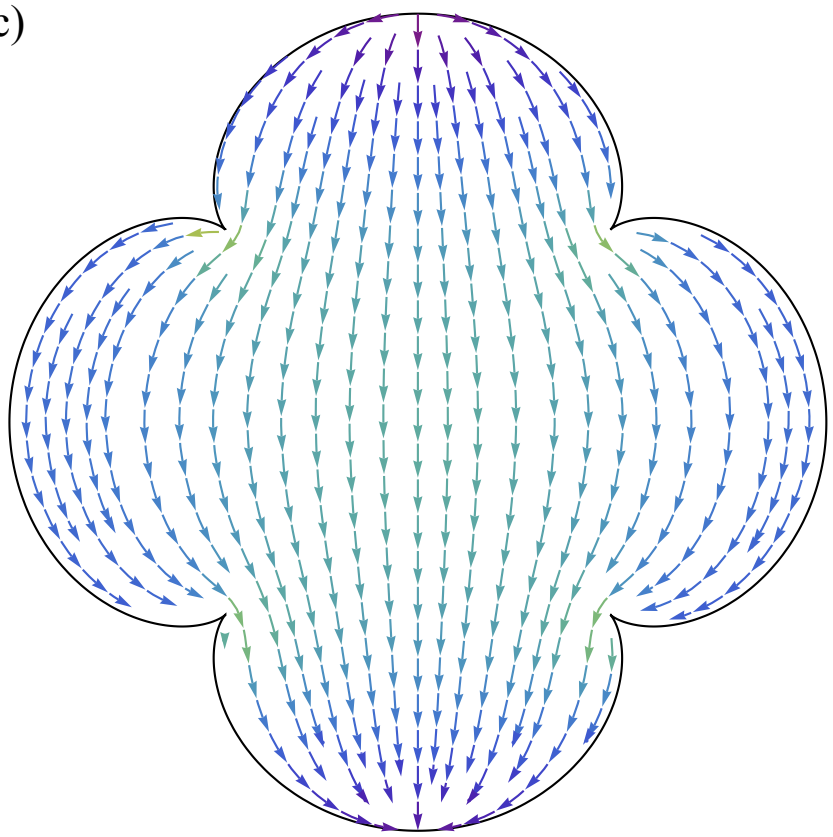

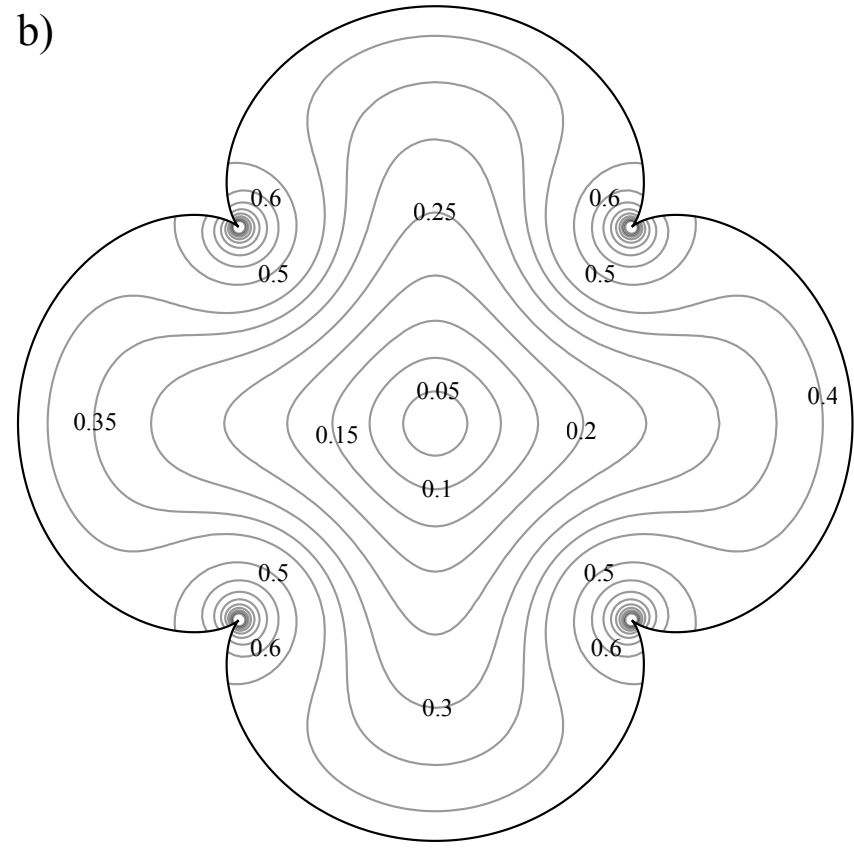

d)

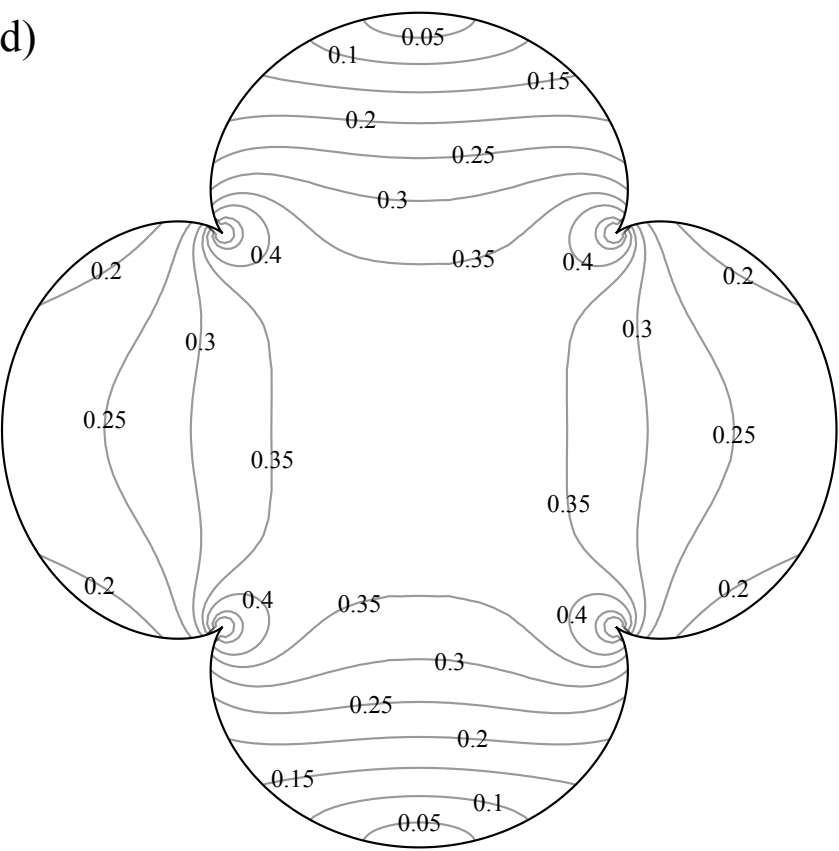

FIGURE 5: Shear stress vector fields and shear stress magnitude contour plots in epicycloid-shaped crosssection evaluated by CVBEM considering (a-b) pure torsion $\left(M_{z}=1\right)$ and $(\mathrm{c}-\mathrm{d})$ shear along the $x$ direction $\left(T_{x}=1\right)$. 